\title{
Custos de ajustes de investimento incluindo custos fixos e descontos por quantidade com aplicações a empresas brasileiras
}

\author{
Bruno Miranda Henrique \\ Mestrando - Programa de Pós-Graduação em Administração - Universidade de Brasília (UnB) \\ Endereço: Campus Universitário Darcy Ribeiro, Prédio da FACE - Brasília/DF \\ CEP: 70910-900 - E-mail: brunomhenrique@hotmail.com
}

Recebido: 25/08/2016. Aceite: 26/06/2017.

\section{Resumo}

Custos de investimento são valores pagos pela organização para ajustar seu estoque de capital produtivo. Tais custos são de difícil mensuração direta, sendo analisados agregadamente pelo valor da firma. Assim, o objetivo deste trabalho é equacionar as curvas de custos de ajustes de investimento a partir de dados de balanço de empresas brasileiras. São estudadas as formas gerais mais comuns na literatura, como a quadrática em relação ao investimento, e propostas componentes da equação, incluindo descontos por quantidade e custos fixos. São usadas regressões lineares para adaptar as curvas aos dados empíricos de empresas selecionadas na BOVESPA. Com isso, demonstra-se que os custos de ajustes de investimento nem sempre são explicados apenas por componentes quadráticos no investimento. Nestes casos, a curva de ajuste de investimentos torna-se melhor adaptada a dados empíricos quando considerados descontos por quantidade e custos fixos, além do tradicional componente quadrático com relação aos investimentos. Finalmente, é proposta e analisada empiricamente uma equação simplificada de retorno do investimento em função do $q$ de Tobin.

\section{Palavras-Chave}

Investimento. Custo de ajuste. Preço sombra. Valor da firma.

\begin{abstract}
Investment costs are paid by organizations adjusting their productive capital stock. Those costs are hardly directly measurable, being analyzed aggregately through the value of the firm. Therefore, the goal of this work is to find equations for the investment adjustment costs using balance sheets data from Brazilian companies. Common general forms of the curves found in the literature are studied, like the quadratic relation to the investment, and equation components are proposed, including quantity discounts and fixed costs. Linear regressions are used to adapt the curves to the empirical data of companies selected from BOVESPA. Thus, it is shown that investment adjustment costs are not always explained just with the quadratic component with respect to the investments. In those cases, the adjustment costs curve is

- O autor gostaria de agradecer o esmero do trabalho dos editores e revisores, que fizeram críticas e sugestões valiosas para garantir a correção e a qualidade desta publicação, contribuindo até mesmo na seleção de proxies corretas no exercício empírico.
\end{abstract}


better fitted to empirical data when fixed costs and quantity discounts are considered, besides the traditional quadratic component of the investments. Finally, a simplified equation for the investment return is proposed and empirically analyzed as a function of Tobin's $q$.

\section{Keywords}

Investment. Adjustment cost. Shadow price. Value of the firm.

\section{JEL Classification}

C13. C51. D24.

\section{Introdução}

Uma organização busca aumentar seu valor através de investimentos que aumentam seu lucro operacional. Entretanto, aumentar o capital produtivo, isto é, investir objetivando lucratividade, significa incorrer em custos adicionais. Os custos de investimento, ou de ajustes de investimento, são valores pagos pela organização para ajustar seu estoque de capital produtivo, aumentando-o ou diminuindo-o. Este estudo caracteriza formas teóricas das curvas de ajustes de investimentos, incluindo custos fixos e descontos por quantidade, trazendo exemplos empíricos de duas empresas brasileiras.

A decisão de investir da firma é afetada pela incerteza sobre o futuro do mercado. Pindyck (1988) investiga o valor da firma sob decisões incrementais de investimento sobre sua capacidade. Porém a valoração da firma deve considerar o preço de não realizar o investimento ou mesmo adiá-lo, como tratado por McDonald e Siegel (1986). Tais investimentos são realizados sob um custo, função da mudança no estoque de capital produtivo. Gould (1968) estuda este custo do investimento, aproximando-o por uma função específica o suficiente para capturar efeitos empíricos. Alguns custos práticos incorridos quando de investimentos sobre capital produtivo são de contratações, estocagem e instalação de maquinário, para citar exemplos.

Os custos de ajuste são centrais no estudo de investimentos corporativos. Abel e Eberly (1994), por exemplo, baseiam todo seu modelo de regimes de investimento na forma da curva de ajuste de custos. Por sua vez, o estudo de Cooper e Haltiwanger (2006) afirma ser vital seu entendimento para avaliar políticas de investimento dinâmicas, taxações e fatores de difícil medição, como os custos de entrega e instalação. Outros estudos 
igualmente importantes, como Barnett e Sakellaris (1998) e Bolton et al. (2011), relacionam a curva de custos de ajuste e investimentos ao $q$ de Tobin, creditado a Tobin (1969).

Dada a importância dos custos de ajuste em investimentos, importa estudar a forma e as características dessa curva. Comumente a literatura atribui-lhe uma forma convexa, como argumenta Abel e Eberly (1994). Entretanto alguns autores, como Hamermesh e Pfann (1996), afirmam que as curvas de custos de ajustes de investimento podem assumir outras formas. Sendo assim, são buscados componentes diferentes das convexas em custos de investimento. Uma contribuição especial a esses custos pode ser encontrada na literatura de descontos por quantidade, revisada por Munson e Rosenblatt (2009).

Os custos dos investimentos são deduzidos dos lucros operacionais da firma para se obter uma estimativa de valor da mesma. Contudo, modelar os custos de ajustes de investimento não é simples, uma vez que envolve fatores de difícil mensuração, como argumenta Cooper e Haltiwanger (2006). Além disso, a literatura acerca de custos de ajustes de investimento, a exemplo de Abel e Eberly (1994), relaciona-se intimamente com a do $q$ de Tobin, outra quantidade de difícil mensuração prática. $\mathrm{O} q$ de Tobin, chamado "preço sombra", não é diretamente observado no mercado corporativo, e sua quantificação é importante tópico de pesquisas empíricas.

Eminentes referências da literatura modelam o valor da firma usando lucros operacionais e custos de investimento, tais como Abel e Eberly (1994) e Cooper e Haltiwanger (2006). Sendo assim, uma função geral de custos de ajustes de investimento, de comportamento e forma bem definidos, é desejável. Entretanto, essa é uma problemática de difícil solução devido aos inúmeros fatores contribuindo para estes custos. Trabalhos teórico-empíricos, como o de Lundgren e Sjöström (2001), tentam dar uma forma geral a tais custos, mas mesmo estes autores concluem que os custos podem assumir múltiplas formas numa mesma firma, a depender do tipo de investimento. Outros estudos, como a avaliação empírica de Groth e Khan (2010), concluem que a problemática dos custos de ajustes de investimento é sujeita a muitos fatores ainda não explicados.

O presente trabalho objetiva analisar os custos de ajustes de investimento, de forma agregada ao valor da firma. O objetivo principal é caracterizar matematicamente as equações das curvas de custo de ajustes 
de investimento, incluindo custos fixos e descontos por quantidade de investimentos. Para ilustrar, exemplos empíricos estimam tais curvas para duas empresas brasileiras. Observa-se que os descontos por quantidade são ricamente explorados como um tema central de pesquisas. Contudo, este trabalho objetiva introduzir simplificadamente a teoria de descontos por quantidade em estimativas das funções de custos de investimento. Finalmente, usando resultados de estimativas das curvas de custos de ajustes, este trabalho analisa empiricamente a proposta de estudo de retornos de investimento trabalhada por Abel e Eberly (1994), enriquecendo a teoria com um exemplo de retornos não tratado pelos autores.

Para alcançar os objetivos principais deste trabalho, são formuladas algumas hipóteses sobre as formas gerais das curvas de ajustes de investimentos. Apesar da comum assunção na literatura de convexidade de custos com relação aos investimentos, investiga-se a precisão dessas curvas em dois casos reais. Em especial, busca-se a presença significativa de componentes de descontos nas curvas de custos de investimentos.

\section{Referencial teórico}

Demonstrando a presença dos custos de investimento a partir do modelo de valoração da firma, Gould (1968) estuda a dinâmica dos investimentos à luz de seus custos. Esse autor afirma que a natureza desses custos está nos fatores encarecedores do investimento. São custos internos e externos indistintamente considerados sob uma mesma equação. Em seu artigo, Gould (1968) questiona como seria uma especificação matemática para uma curva de custos de ajuste em função do investimento realizado. Tal texto então assume uma forma quadrática de simples tratamento matemático e que se aproxima de considerações empíricas.

Conforme descrito acima, o texto de Gould (1968) toma uma curva convexa para a função de custos de investimento. A convexidade é assumida em muitos textos, a exemplo de Abel e Eberly (1994) e Bolton et al. (2011). Contudo, Rothschild (1971) chama a atenção para o peso da assunção de convexidade para os resultados de dinâmicas de investimentos na literatura. Sendo assim, esse autor propõe uma forma não convexa para a curva de custos de ajuste, estudando as implicações para os investimentos da firma. 
O estudo de custos de investimentos em Hamermesh e Pfann (1996) é realizado sob a ótica de ajustes de fatores produtivos da firma. Tal estudo especifica formas lineares, irregulares e convexas para as curvas de custos, considerando inclusive possíveis assimetrias em torno do investimento zero. ${ }^{1}$ Também buscando outras possibilidades à forma da curva de custos de ajuste de investimento, Lundgren e Sjöström (2001) propõem uma especificação flexível o suficiente para acomodar cinco formas, dentre elas a forma côncava e uma equação constante, de apenas custos fixos.

Conforme Lundgren e Sjöström (2001) e Hamermesh e Pfann (1996), a tradicional forma convexa da função de custos de investimento não se adapta a diversos casos empíricos. Assim, Cooper e Haltiwanger (2006) estudam algumas formas além da convexa e concluem, com dados empíricos e simulados, que nenhuma delas isoladamente se adapta a casos reais. Aqueles autores apontam então para uma função de características mistas, côncavas e convexas. Outro estudo empírico que conclui pela dificuldade em dar forma aos custos de ajuste é o de Groth e Khan (2010). Analisando indústrias americanas, os autores usam uma abordagem desagregada de estimação dos custos, reportando que muitos componentes dos mesmos permanecem sem explicação direta.

A função de custos de ajustes de investimento é central na teoria desenvolvida por Abel e Eberly (1994). Os autores consideram os custos fixos apenas como uma descontinuidade na curva de custos de ajuste. O presente trabalho, no entanto, inclui os custos fixos explicitamente na curva teórica. Abel e Eberly (1994) derivam ainda uma forma simplificada de retorno de investimentos como uma função do $q$ de Tobin e do investimento ótimo escolhido. Em sua teoria, Abel e Eberly (1994) tecem conclusões fundamentais sobre regimes de investimentos com base na forma da curva de custos dos mesmos e do $q$ de Tobin. Os resultados a seguir exemplificam formas empíricas de tais curvas em $q$, estendendo a teoria para incluir descontos por quantidade. Outra contribuição de grande valor de Abel e Eberly (1994) é a proposição de duas formas de medir os preços sombra. Tais preços são relacionados aos custos do investimento em Barnett e Sakellaris (1998).

Conforme descrito anteriormente, o presente trabalho propõe incluir nos custos do investimento os descontos por quantidade adquirida. Trata-se de

1 Especificamente o artigo de Hamermesh e Pfann (1996) trata de contratações, mas seu tratamento dos custos de ajuste tem aplicação análoga a investimentos, principalmente quanto à forma das curvas. 
uma tradição nos negócios, uma expectativa de o comprador ver o preço do produto diminuído devido ao montante negociado com o vendedor, como introduzem Munson e Rosenblatt (2009). O assunto começou a ser abordado academicamente nos anos 70 , mas foi nos anos 80 que a perspectiva passou ao vendedor, que tentava estimular a demanda anual oferecendo descontos por quantidade. Munson e Rosenblatt (2009) revisam a literatura no assunto de descontos por quantidade, tabulando as fontes da literatura em perspectivas do comprador, do vendedor e mistas. Estes autores trazem ainda um estudo empírico através de entrevistas com 39 representantes de companhias americanas. Seu estudo aponta fatores que levam à implantação de políticas de descontos por quantidade negociada.

O desconto por quantidade influencia diretamente na decisão de investir, conforme argumentam Schotanus et al. (2009). Tal artigo propõe uma função que descreve os descontos de acordo com preços e quantidades negociadas. Apesar das limitantes assunções sobre esta função, os autores verificam empiricamente sua adaptabilidade a programas de descontos reais. Um aprofundamento nos aspectos integrantes de descontos por quantidade em compras é encontrado em Li e Huang (1995). Por sua vez, Hwang et al. (1990) detalham matematicamente um modelo para computar custos totais na presença de descontos por quantidade. $O$ presente estudo inclui uma componente de descontos por quantidade nos custos totais de ajuste dos investimentos. Sendo uma componente significativa no equacionamento dos custos, trata-se de uma prática de mercado que altera a forma das curvas de custos e, com isso, deve alterar o planejamento de uma empresa. Importantes estudos, tais como Gould (1968), Abel e Eberly (1994), Hamermesh e Pfann (1996) e Lundgren e Sjöström (2001), tratam os custos de investimentos sem considerações sobre os descontos por quantidade.

Mesmo sujeitos a muitos fatores de influência em sua computação, os custos de ajustes de investimento são objetos de análises empíricas. Groth e Khan (2010) propõem medi-los de forma desagregada ao valor da firma. Estes autores medem valores baixos para investimentos em manufaturas americanas, sendo os investimentos muito responsivos ao "preço sombra". Este termo remonta ao texto de Tobin (1969), que introduz uma quantidade $q$ em sua teoria monetária de análise de mercado. O $q$ de Tobin encontra importantes aplicações em finanças corporativas, como no gerenciamento de riscos assumidos por empresas sob restrições de investimento e financiamento, tratado por Aldrighi e Bisinha (2010) e Bolton et al. (2011). 


\section{Metodologia}

Passa-se à construção do modelo funcional de relação entre valor da firma, investimentos, custos e "preços sombra". As equações são construídas em função da curva de custos de ajuste, buscando formalizar custos fixos e descontos por quantidade separadamente. Seguem abaixo as especificações e modelagens.

\subsection{Modelo básico}

Seja a função de lucro operacional da empresa denotada por $\Pi$. Assim como Cooper e Haltiwanger (2006), assume-se que seja uma função linearmente homogênea com relação ao estoque de capital de produção $K$. Tal função é sujeita a uma variável aleatória $A$ representando choques ou variações na produtividade ou trabalho da firma:

$$
\Pi(K, A)=A K^{\theta}
$$

onde $\theta$ é um grau de homogeneidade qualquer. $A$ pode ser gerada por um processo estocástico modelando variações na tecnologia, custos trabalhistas ou processos judiciais, por exemplo. Usando $W$ para denotar um processo de Wiener padrão, a variável aleatória $A$ segue a difusão temporal

$$
d \epsilon=\mu(\epsilon) d t+\sigma(\epsilon) d W
$$

em que $\mu$ e $\sigma$ representam respectivamente o drift e o desvio padrão do processo.

A problemática de investimentos sob incertezas e custos trata da escolha ótima de incremento ou decremento do capital produtivo K. Assume-se que esse capital evolui no tempo de acordo com o processo abaixo, onde $\delta$ é uma taxa de depreciação qualquer e $I_{t}$ é o investimento no período:

$$
d K_{t}=\left(I_{t}-\delta K_{t}\right) d t
$$

Observa-se que o investimento é modelado como uma quantidade de capital a ser incrementada ou decrementada do estoque de capital atual. Sendo assim, o investimento da firma no período presente $I_{t}$ é totalmente 
acrescido ao capital de produção no próximo período, sem perdas ou tempo de instalação. Eventuais perdas no investimento são consideradas custos e, portanto, estão incluídas na função de custos de ajuste, modelada a seguir. Portanto $I_{t}=K_{t+1}-K_{t}$ e a Equação (3) torna-se

$$
d K_{t}=\left[K_{t+1}-K_{t}(\delta+1)\right] d t
$$

O valor da firma num tempo $t$ é o valor esperado da diferença entre o lucro operacional e os custos totais de investimento, descontado de uma taxa $r$. Para os fins deste trabalho, os custos de investimento serão explicitados em custos de ajustes propriamente ditos, custos fixos e eventuais descontos por quantidade de compras. Tal é feito para estudar o comportamento do custo total de investimento de acordo com cada uma dessas componentes. Portanto, o modelo assume a possibilidade de funções de ajuste de custo de qualquer forma além da tradicional convexa. Além disso, o modelo possibilita reversibilidade, denotada por $I_{t}<0$, isto é, $K_{t}>K_{t+1}$. Contudo, os casos de descontos por quantidade são tratados apenas para $I_{t}>0$. Logo, não são considerados descontos por quantidade quando a firma diminui capital de produção.

\subsection{Valor da firma}

A forma geral do valor da firma no tempo $t$ é dada pelo valor trazido a presente dos fluxos contínuos no tempo dos lucros operacionais descontados dos custos totais de investimento. Então a firma escolhe o investimento $I_{t}$ tal que:

$$
\begin{aligned}
V\left(K_{t}, \epsilon_{1, t}, \epsilon_{2, t}\right) & \\
& =\max _{I_{t+s}} \int_{0}^{\infty} E_{t}\left[\Pi\left(K_{t+s}, \epsilon_{1, t+s}\right)-C\left(I_{t+s}, K_{t+s}, \epsilon_{2, t+s}\right)-F_{t+s}\right. \\
& \left.+\xi\left(I_{t+s}\right)\right] e^{-r s} d s
\end{aligned}
$$

A Equação (5) ressalta as três componentes do custo total de investimentos: (1) uma função de ajuste de custos $C\left(I_{t+s}, K_{t+s}, \epsilon_{2, t+s}\right)$, (2) os cus- 
tos fixos denotados por $F_{t+s}$ que podem variar no tempo, ${ }^{2}$ e (3) $\xi\left(I_{t+s}\right)$ representando o desconto conseguido devido ao volume do investimento. Observa-se que este valor é um retorno para a firma, isto é, um valor recebido apesar do incremento real no capital produtivo. Isso significa que ao realizar um incremento em de $K_{t}$ acordo com a Equação (4), a firma teoricamente investe $I_{t}$, atingindo o capital $K_{t+1}$, porém efetivamente paga um valor com desconto $\left(I_{t}-\xi\left(I_{t}\right)\right)$.

É importante ressaltar a aleatoriedade presente nos termos do valor da firma. Conforme modelado anteriormente na Equação (1), o lucro operacional está sujeito a aleatoriedades diversas. Por sua vez, o custo de ajuste de investimentos sujeita-se a outras aleatoriedades, por exemplo, variações em frete, custo de instalação, interrupções na produção. Apesar de ambas as aleatoriedades serem difusões descritas pela Equação (2), elas seguem processos distintos entre si na Equação (5).

Claramente nenhum dos custos da Equação (5) estará presente na valoração caso a firma decida não investir. Neste caso, o valor da firma é apenas o valor esperado de seu lucro operacional com o capital produtivo atual, descontado a uma taxa $r$. Para incluir essa possibilidade no modelo da Equação (5), usa-se a função indicadora abaixo:

$$
i(I)= \begin{cases}1 & \text { para } I \neq 0 \\ 0 & \text { para } I=0\end{cases}
$$

e portanto a Equação (5) toma a forma

$$
\begin{aligned}
V\left(K_{t}, \epsilon_{1, t}, \epsilon_{2, t}\right) & \\
& =\max _{I_{t+s}} \int_{0}^{\infty} E_{t}\left\{\Pi\left(K_{t+s}, \epsilon_{1, t+s}\right)-i\left(I_{t+s}\right)\left[C\left(I_{t+s}, K_{t+s}, \epsilon_{2, t+s}\right)+F_{t+s}\right.\right. \\
& \left.\left.-\xi\left(I_{t+s}\right)\right]\right\} e^{-r s} d s
\end{aligned}
$$

Observa-se a concisão na notação usada na Equação (7). Tal equação representa o valor da firma sob a decisão de investir ou não, modelada pela

2 A rigor, os custos fixos dependem do sinal de $I$, isto é, os custos fixos incorridos em investimentos podem ser diferentes dos custos fixos ao se realizar desinvestimentos. Este texto considera-os iguais apenas para simplificar notações e manter concisas as equações. 
função indicadora na Equação (6). Este modelo dispensa o uso de equações diferentes para cada decisão da firma, simplificando os desenvolvimentos que se seguem.

O problema de maximização da Equação (7), e condições dadas nas Equações (2) e (4), é especificado numa variável incremental $s$ representando a continuidade temporal. Contudo, o trabalho empírico aqui proposto como exemplos dos casos teóricos sugere o uso de um modelo especificado em períodos discretos, uma vez que os dados são coletados de acordo com uma periodicidade. Portanto, pode-se modelar o investimento que maximiza o valor da firma de acordo com o valor atual acrescido do valor esperado da firma nos próximos períodos, descontado a uma taxa $(1-r)$ :

$$
\begin{aligned}
V\left(K_{t}, \epsilon_{1, t}, \epsilon_{2, t}\right) & \\
= & \max _{I}\left\{\Pi\left(K_{t}, \epsilon_{1, t}\right)-i\left(I_{t}\right)\left[C\left(I_{t}, K_{t}, \epsilon_{2, t}\right)+F_{t}-\xi\left(I_{t}\right)\right]\right. \\
& \left.+(1-r) E_{t}\left[V\left(K_{t+1}, \epsilon_{1, t+1}, \epsilon_{2, t+1}\right)\right]\right\}
\end{aligned}
$$

que, considerando períodos incrementais no tempo, assume a forma de Bellman abaixo:

$$
\begin{array}{r}
V\left(K, \epsilon_{1}, \epsilon_{2}\right)=\max _{I}\left\{\Pi\left(K, \epsilon_{1}\right)-i(I)\left[C\left(I, K, \epsilon_{2}\right)+\right.\right. \\
\left.F-\xi(I)]+(1-r) E_{t}\left[\frac{d V}{d t}\right]\right\}
\end{array}
$$

A Equação (8) tem sua demonstração formal relegada ao Apêndice. De acordo com esta equação, a firma escolhe o investimento ótimo que maximiza seu valor, função de uma componente incremental de valor no futuro. O problema na escolha de investimentos é maximizar o lucro operacional descontado de um custo de realizar o investimento e somado à esperança de incremento ao valor da empresa, denotada por $E_{t}[d V / d t]$. Além disso, a notação da Equação (8) evidencia a presença de processos estocásticos distintos na valoração da empresa. Neste ponto, este texto diferencia-se na notação de Abel e Eberly (1994), que não considera aleatoriedades específicas em sua função de custos de ajuste aumentada, considerando-as apenas no lucro operacional. 
É importante ressaltar o caráter instantâneo Equação (8). Observa-se que até a Equação (7) todo o tratamento matemático foi feito com o tempo denotado pelo subscrito $t$, pois admite-se que o valor da firma, bem como as variáveis da qual ele depende, deve variar com o tempo. No entanto, a Equação (8) considera a variação do valor da firma no tempo por meio do termo incremental $E_{t}[d V / d t]$. Isto é, num tempo infinitesimal $d t$, a firma tem seu valor variando no valor esperado da taxa $d V / d t$.

Para dar tratamento empírico ao modelo funcional da Equação (8), é necessário explicitar o termo incremental do valor da firma. Para tanto, usa-se o Lema de Itô para dois processos estocásticos que, para a Equação (2) e uma função escalar $V$ diferenciável duas vezes, deriva a conhecida relação:

$$
\begin{aligned}
d V\left(\epsilon_{1, t}, \epsilon_{2, t}, t\right) & \\
= & \left(\frac{\partial V}{\partial t}+\frac{\partial V}{\partial \epsilon_{1}} \mu_{1}+\frac{\partial V}{\partial \epsilon_{2}} \mu_{2}+\frac{1}{2} \frac{\partial^{2} V}{\partial \epsilon_{1} \partial \epsilon_{2}} \sigma_{1} \sigma_{2} \rho\right) d t+\frac{\partial V}{\partial \epsilon_{1}} \sigma_{1} d W_{1} \\
& +\frac{\partial V}{\partial \epsilon_{2}} \sigma_{2} d W_{2}
\end{aligned}
$$

em que $\rho$ é a correlação entre os processos de Wiener $W_{1}$ e $W_{2}$. Usando-se a Equação (3) na forma $d t=d K /(I-\delta K)$ e o fato de que o valor esperado de um processo de Wiener $d W$ é zero, tem-se

$$
d V=\left(\frac{\partial V}{\partial K}(I-\delta K)+\frac{\partial V}{\partial \epsilon_{1}} \mu_{1}+\frac{\partial V}{\partial \epsilon_{2}} \mu_{2}+\frac{1}{2} \frac{\partial^{2} V}{\partial \epsilon_{1} \partial \epsilon_{2}} \sigma_{1} \sigma_{2} \rho\right) d t
$$

que substituído na Equação (8) resulta em

$$
\begin{aligned}
V\left(K, \epsilon_{1}, \epsilon_{2}\right) & =\max _{I}\left\{\Pi\left(K, \epsilon_{1}\right)-i(I)\left[C\left(I, K, \epsilon_{2}\right)+F-\xi(I)\right]\right. \\
& \left.+(1-r)\left[\frac{\partial V}{\partial K}(I-\delta K)+\frac{\partial V}{\partial \epsilon_{1}} \mu_{1}+\frac{\partial V}{\partial \epsilon_{2}} \mu_{2}+\frac{1}{2} \frac{\partial^{2} V}{\partial \epsilon_{1} \partial \epsilon_{2}} \sigma_{1} \sigma_{2} \rho\right]\right\}
\end{aligned}
$$

Ressalta-se a presença da definição do $q$ de Tobin na Equação (9):

$$
q=\frac{\partial V}{\partial K}
$$


que pode ser medido empiricamente através de um importante resultado de Abel e Eberly (1994). Os autores mostram que se as funções de lucro operacional e de custos de investimentos forem linearmente homogêneas em $I$ e $K$, o $q$ marginal pode ser medido pelo $q$ médio. Tal aproximação continua válida mesmo na presença de custos fixos e, com isso,

$$
q=\frac{V}{K}
$$

Observa-se que as três últimas parcelas da Equação (9) são independentes do investimento $I$ da maximização e variam de acordo com processos estocásticos de forma definida na Equação (2). Os efeitos aleatórios ao investimento e capital produtivo dessas parcelas podem ser agregados numa única função definida como:

$$
Z\left[V\left(K, \epsilon_{1}, \epsilon_{2}\right)\right]=\frac{\partial V}{\partial \epsilon_{1}} \mu_{1}+\frac{\partial V}{\partial \epsilon_{2}} \mu_{2}+\frac{1}{2} \frac{\partial^{2} V}{\partial \epsilon_{1} \partial \epsilon_{2}} \sigma_{1} \sigma_{2} \rho
$$

Usando as definições das Equações (10) e (12) e $I_{t}=K_{t+1}-K_{t}$ no modelo funcional da Equação (9), tem-se uma expressão final com quantidades explicitadas para um trabalho empírico:

$$
\begin{aligned}
V\left(K, \epsilon_{1}, \epsilon_{2}\right)= & \max _{I}\left\{\Pi\left(K, \epsilon_{1}\right)-i(I)\left[C\left(I, K, \epsilon_{2}\right)+F-\xi(I)\right]\right. \\
& \left.+(1-r)\left[q\left(K_{t+1}-K_{t}-\delta K_{t}\right)+Z\left[V\left(K, \epsilon_{1}, \epsilon_{2}\right)\right]\right]\right\}
\end{aligned}
$$

A Equação (13) calcula o valor atual da firma em função de um investimento $I_{t}=K_{t+1}-K_{t}$ admitindo-se que a firma faz a escolha para maximizar seu valor. Esta decisão pode ser de não realizar investimentos, contemplada na possibilidade $i(0)=0 . \mathrm{Na}$ Equação (13) o valor da firma é então composto por três termos. O primeiro é o lucro operacional atual da firma, antes do investimento em capital de produção. O segundo termo representa os custos de ajuste de investimentos, quando realizados no período corrente. O terceiro termo é o valor marginal da firma por unidade de capital, ponderado por investimento e depreciação, acrescido de um choque de aleatoriedades. Tais aleatoriedades, devidas aos processos estocásticos 
presentes no lucro operacional e nos custos de ajuste de investimentos, são agregadas no termo representado pela Equação (12).

Caso a firma escolha realizar um investimento $I$ sobre capital de produção $K$, ela deve fazê-lo buscando a maximização da Equação (13). Neste caso $i(I)=1$ e os custos de ajuste do investimento ótimo escolhido, denotado por $I^{*}$, serão:

$$
\begin{aligned}
{\left[C\left(I^{*}, K, \epsilon_{2}\right)+F-\right.} & \left.\xi\left(I^{*}\right)\right] \\
& =\Pi\left(K, \epsilon_{1}\right)-V\left(K, \epsilon_{1}, \epsilon_{2}\right)+ \\
& (1-r)\left[q\left(K_{t+1}-K_{t}-\delta K_{t}\right)+Z\left[V\left(K, \epsilon_{1}, \epsilon_{2}\right)\right]\right]
\end{aligned}
$$

Esta equação possibilita estimativas dos custos de ajustes de investimentos diretamente através de dados empíricos. Nota-se o caráter estático no tempo da Equação (14), possibilitando que as variáveis independentes do lado direito da equação sejam tomadas como amostras nos balanços e demonstrativos de empresas selecionadas para estudos práticos. Contudo, alerta-se para a cautela necessária na aplicação destas análises, uma vez que na prática as curvas de ajustes de investimentos podem variar com o tempo. O tratamento instantâneo da eq. (14) é usado apenas para ilustrar como os custos totais de investimentos variam conforme variam as variáveis independentes do lado direito da equação, mantendo-se constante no tempo a forma da curva.

\subsection{Retorno do investimento}

Abel e Eberly (1994) sugerem uma nova medida do retorno dos investimentos, removendo da equação de Bellman os termos independentes deste investimento. Seguindo este raciocínio, constrói-se uma nova equação a partir da Equação (13), mantendo apenas os termos dependentes do investimento $I$. Sendo assim, após a escolha de um $I^{*}$ que satisfaça a Equação (13), equaciona-se uma nova função desprezando-se os termos independentes de $I^{*}$ :

$$
\psi\left(q, I^{*}, K\right)=q(1-r)\left(K_{t+1}-K_{t}\right)-i\left(I^{*}\right)\left[C\left(I^{*}, K, \epsilon_{2}\right)+F-\xi\left(I^{*}\right)\right]
$$


A Equação (15) é uma forma de representar o valor da firma considerando-se apenas a influência do $q$ de Tobin, mantendo-se fixos investimento ótimo e capital produtivo. Uma vez que ela ignora o lucro operacional da firma e outros componentes aleatórios independentes de $I^{*}$, trata-se de uma medida especial de retorno do investimento, independente inclusive do custo fixo de investimento. Abel e Eberly (1994) usam de semelhante equação para determinar regimes de investimentos em função do $q$ de Tobin. Contudo, a formulação matemática de Abel e Eberly (1994) deixa implícita a presença de custos fixos. Os autores tratam os custos fixos como caso especial de descontinuidade da função de ajuste de custos quando $I=0$. Como visto na Equação (15), o presente trabalho opta por explicitar os custos fixos em $F$, dispensando uma formulação vinculada a descontinuidades em $C\left(I, K, \epsilon_{2}\right)$.

Abel e Eberly (1994) argumentam que a empresa selecionará um investimento ótimo $I^{*}$ que maximize a Equação 15 para o custo marginal do investimento igualar-se ao benefício marginal do mesmo. Sendo assim, a empresa seleciona $I^{*}$ tal que

$$
\frac{\partial}{\partial I}\left[C\left(I^{*}, K, \epsilon_{2}\right)+F-\xi\left(I^{*}\right)\right]=q
$$

Sendo assim, após especificar uma função para os custos de ajuste de investimentos, caso ela seja diferenciável em todos os pontos, é possível calcular o custo marginal do investimento, igualando-o ao $q$ de Tobin. Isolando $I$ e substituindo na Equação (15), tem-se uma medida especial de retorno do investimento em função apenas de $q$ e $K$. Portanto, a medida $\psi(q, K)$ possibilita avaliar regimes de investimento em função de $q$ ignorando-se o lucro operacional da empresa dado na Equação (1) e fixando-se o capital produtivo.

Os próximos parágrafos destinam-se a detalhar a modelagem matemática das três componentes do custo ressaltadas na Equação (13). A análise empírica deste texto busca exemplificar a presença destas componentes nos custos de investimentos. 


\subsection{Custos de ajuste e custos fixos}

Os custos de ajuste propriamente ditos, denotados $C($ ) como nas equações, são comumente assumidos como função do investimento $I$ e do estoque de capital produtivo $K$, como descrevem Abel e Eberly (1994). Entretanto, o presente trabalho adota o raciocínio de Cooper e Haltiwanger (2006), entendendo que os custos também são função de uma componente aleatória $\epsilon_{t}$. Esta componente aleatória diz respeito, por exemplo, a negociações com fornecedores ou choques de disponibilidade ou de demanda pelo recurso no mercado. O modelo de difusão desta aleatoriedade é dado na Equação (2). Portanto, tais custos são representados por $C\left(I, K, \epsilon_{2}\right)$.

A literatura comumente assume uma função convexa para os custos de ajuste. Abel e Eberly (1994) baseiam suas conclusões nessa premissa, sem uma formulação matemática explícita para tal função. Seguindo a convexidade, estudos teóricos como Gould (1968) e Hamermesh e Pfann (1996), e trabalhos empíricos e simulados como Cooper e Haltiwanger (2006) e Bolton et al. (2011), equacionam uma forma quadrática para os custos de ajuste. Seguindo os modelos propostos por esses autores, define-se uma componente convexa em I para os custos de ajuste, na forma

$$
C(I, K)=\frac{\gamma}{2} \frac{I^{2}}{K}
$$

onde $\gamma$ é um parâmetro constante, usado apenas para flexibilizar a forma da parábola. A literatura usa uma função desse tipo por seu conhecido tratamento econométrico, conforme Rothschild (1971). Além disso, como afirmado em Lundgren e Sjöström (2001), a função quadrática adapta-se a muitos estudos empíricos. Como exceções, alguns resultados empíricos adiante mostram que a curva da Equação (17) nem sempre é a mais adequada para modelar o custo de investimento de empresas brasileiras. Outras componentes, como funções de descontos por quantidade, são possíveis e significativas.

Conforme descrito anteriormente, o presente estudo propõe uma componente aleatória aos custos de investimentos. Sendo assim, os custos de ajuste de investimentos devem ser equacionados como:

$$
C\left(I, K, \epsilon_{2}\right)=\frac{\gamma}{2} \frac{I^{2}}{K}+\epsilon_{2}
$$


A Equação (17) é simétrica com relação a $I=0$. Sendo assim, os custos serão os mesmos tanto para quantidades $I$ investidas no capital de produção quanto para quantidades I desinvestidas do mesmo. Com isso admitese que os custos de ajuste são os mesmos tanto na compra de maquinário como na venda, por exemplo. Naturalmente pode-se especificar formas diferentes de $C\left(I, K, \epsilon_{2}\right)$ para investimentos $(I>0)$ e desinvestimentos $(I<0)$. A forma escolhida na Equação (17) reduz notações e facilita a representação de resultados e formas, sendo geral o suficiente para os propósitos deste trabalho. Formas assimétricas são tratadas detalhadamente em Hamermesh e Pfann (1996). Apesar de comumente os custos de ajuste serem especificados na forma da Equação (17), outras funções de $I$ e $K$ são igualmente possíveis. Em seu tratamento empírico, este texto propõe formas complementares à forma da Equação (17), adaptando-se melhor aos dados coletados de duas empresas brasileiras, usadas como exemplos. Com isso, são testadas formas de equações como a (17) e também formas contendo descontos por quantidade.

Por sua vez, os custos fixos são modelados como $F$, constantes em relação ao investimento. $\mathrm{O}$ modelo admite que os custos fixos podem variar com o tempo, mas não com o nível do investimento. Caso tais custos variem com o investimento, eles são assumidos como parte integrante do custo de ajuste $C\left(I, K, \epsilon_{2}\right)$ descrito nos parágrafos anteriores.

Ressalta-se a importância de explicitar os custos fixos nos custos totais de investimentos da firma. Dessa forma, os custos fixos não dependem das especificidades do custo de ajuste $C\left(I, K, \epsilon_{2}\right)$, dando liberdade na modelagem de custos. Este tratamento difere da estratégia adotada por Abel e Eberly (1994), que modela o custo fixo como um caso de descontinuidade em 0 na curva de ajuste de investimentos, chamada $C(0, K)$.

\subsection{Descontos}

Finalmente, integrando os custos totais de investimento, existem os descontos por quantidade. A depender do volume de compras, muitos fornecedores oferecem descontos progressivos, função portanto do investimento realizado, como revisam Munson e Rosenblatt (2009). Tais descontos podem variar com o tempo e condições de mercado, como demanda e elasticidade de preços. Tratamentos mais completos para descontos por 
quantidade podem ser encontrados em Hwang et al. (1990) e Li e Huang (1995). Para fins deste estudo, admitem-se descontos variando apenas com o investimento.

Os próximos parágrafos especificam o modelo de descontos usado. Adotase o modelo proposto por Schotanus et al. (2009), dada sua adaptabilidade a programas de descontos empíricos estudados pelos autores. Ao contrário do recomendado por Munson e Rosenblatt (2009), o modelo de descontos por quantidade adotado é contínuo nos preços. As entrevistas conduzidas por Munson e Rosenblatt (2009) concluem que os descontos por quantidade são praticados para limiares de quantidades fixas, isto é, não há continuidade real. Contudo, para fins desse estudo, um modelo contínuo é suficiente para simular sua influência em custos de investimentos. Ademais não há dados facilmente disponíveis agregando todos os programas de descontos dos fornecedores de uma determinada empresa, sendo necessário simular comportamentos. Portanto, dados os objetivos propostos, componentes de desconto nos custos de investimento são evidenciados mesmo adotando-se um modelo simplório de descontos.

Sejam $p$ e $p_{d}$ o preço corrente e o preço com desconto por quantidade acordado de um determinado produto, respectivamente. O preço $p_{d}$ descontado pela quantidade a adquirir é dado por Schotanus et al. (2009) como:

$$
p_{d}(N)=p_{m}+\frac{S}{N^{\eta}}
$$

onde $S$ é um parâmetro fixo de escala, $N$ é a quantidade maior que zero, $p_{m}$ um preço mínimo e $\eta$ uma constante determinada pela política de descontos do vendedor do produto negociado. Aqui, interessa apenas quanto de desconto retorna para a firma, isto é, quanto a firma deixa de pagar devido a uma quantidade acordada. Sendo assim, o retorno para a firma é a diferença entre o que ela teria pago sem os descontos e o pagamento real na presença dos descontos, isto é, $N p-N p_{d}(N)=\xi(N, p)$ :

$$
\xi(N, p)=N p-N\left(p_{m}+\frac{S}{N^{\eta}}\right)=N p-N p_{m}-N \frac{S}{N^{\eta}}
$$

Normalizando os preços em 1 , a quantidade adquirida torna-se o investimento, isto é $I=N p=N 1$. Assumindo que não há um preço mínimo para o início dos descontos, a Equação (20) torna-se: 


$$
\xi(I)=I-\frac{S}{I^{\eta-1}}
$$

válida apenas para $I>0 .^{3}$

Vale ressaltar as adaptações ao modelo inicial de Schotanus et al. (2009) proposto pela Equação (19). Uma delas é o uso de preços normalizados em 1, isto é, modela-se que a aquisição é realizada sempre a um mesmo preço e que este é uma unidade de valor. Com isso, o investimento é representado integralmente por quantas unidades de valor são somadas ao estoque de capital produtivo, isto é, $I=N$. Outra adaptação usada na Equação (21) é a ausência de um preço mínimo para descontos. Neste caso há descontos para qualquer valor de compra.

Na forma proposta da Equação (21), o modelo teria pouca utilidade em estudos de descontos isoladamente. Contudo, o objetivo das simplificações realizadas é introduzir o estudo de descontos nas pesquisas de custos de ajuste de investimentos. Portanto basta um coeficiente $S$ significativo o suficiente para evidenciar a presença dos descontos em curvas empíricas de custos de investimentos.

\subsection{Aproximação quantitativa}

Para aproximar uma relação dos custos de investimento com dados empíricos, coletados para ilustrar a teoria tratada, usam-se regressões lineares, solucionadas por mínimos quadrados. Especificamente são tabulados os valores equivalentes na Equação (14) de acordo com os dados de duas empresas brasileiras selecionadas a seguir. Com isso, os custos de investimento, no lado esquerdo da citada equação, são calculados de forma prática podendo-se dar forma à sua curva. Modelando o lado esquerdo da Equação (14) como especificado pelas Equações (17) e (21), tem-se

$$
\left[C\left(I^{*}, K, \epsilon_{2}\right)+F-\xi\left(I^{*}\right)\right]=\frac{\gamma}{2} \frac{I^{2}}{K}+F-\frac{S}{I^{\eta-1}}
$$

3 Um modelo análogo poderia incluir investimentos negativos. 
que substituída na Equação (14) resulta em:

$$
\begin{aligned}
\frac{\gamma}{2} \frac{I^{2}}{K}+F-\frac{S}{I^{\eta-1}} & =\Pi\left(K, \epsilon_{1}\right)-V\left(K, \epsilon_{1}, \epsilon_{2}\right) \\
& +(1-r)\left[q\left(K_{t+1}-K_{t}-\delta K_{t}\right)+Z\left[V\left(K, \epsilon_{1}, \epsilon_{2}\right)\right]\right]
\end{aligned}
$$

O lado direito da Equação (23) é aproximado por proxies, a menos da componente aleatória $Z\left[V\left(K, \epsilon_{1}, \epsilon_{2}\right)\right]$ dada pela Equação (12), que representa o erro nas estimativas. Logo,

$$
\frac{\gamma}{2} \frac{I^{2}}{K}+F-\frac{S}{I^{\eta-1}}=\text { Custo total de ajuste de investimento }
$$

Portanto, para estimar o custo de investimento como variável dependente, bastam as seguintes variáveis independentes da Equação (23): lucro operacional, valor da firma, capital de produção, $q$ de Tobin, investimentos e taxas de juros e depreciação.

De posse dos custos de investimentos, bastam regressões lineares para adaptá-los em funções bem definidas dadas pelas componentes da Equação (22). Os modelos econométricos de regressão linear selecionados são estritamente equacionados de acordo com as três componentes de custos propostos anteriormente, isto é, custos de ajuste propriamente ditos, custos fixos e descontos por quantidade. A variável dependente é portanto o valor dos custos totais de investimento. Sendo assim, as regressões lineares adaptam os coeficientes $\gamma, S$ e o intercepto $F$ (custo fixo) da Equação (22). O modelo para os custos de ajustes de investimentos tem então a forma geral proposta por:

$$
\frac{\gamma}{2} \frac{I^{2}}{K}-\frac{S}{I^{\eta-1}}+F
$$

Finalmente, com as funções de custos de investimentos aproximadas por regressões lineares, passa-se a analisar o retorno do investimento na forma especial da Equação (15). Para fazê-lo apenas em relação ao $q$ de Tobin, admite-se que as empresas sempre selecionam o investimento ótimo $I^{*}$. 
Neste procedimento são fixados também os respectivos capitais produtivos $K$ em uma média do período dos dados coletados. A Equação (15) pode então ser combinada à Equação (22) para se obter a forma prática:

$$
\psi\left(q, I^{*}, K\right)=q(1-r)\left(K_{t+1}-K_{t}\right)-i\left(I^{*}\right)\left[\frac{\gamma}{2} \frac{I^{2}}{K}+F-\frac{S}{I^{\eta-1}}\right]
$$

A quantificação do $q$ de Tobin nas Equações (23) e (24) é realizada admitindo-se homogeneidade linear para $I$ e $K$ nas funções de lucro operacional e de custos de investimentos. Com essa assunção, $q$ pode ser medido pela Equação (11). Observa-se que o modelo de lucro operacional na Equação (1) atende às condições de homogeneidade linear em $K$. Da mesma forma, todas as componentes dos custos de investimentos usadas neste trabalho são modeladas de forma linear e homogênea em $I$ e em $K$.

\subsection{Dados}

Para ilustrar a aplicação da teoria de custos de ajuste de investimentos como desenvolvida neste trabalho, são selecionados dados de balanço patrimonial de empresas brasileiras de capital aberto, inscritas na Bolsa de Valores de São Paulo (BOVESPA). As empresas escolhidas fazem parte do índice BOVESPA e possuem a relação de balanços patrimoniais amplamente divulgada. A periodicidade dos balanços patrimoniais das empresas é trimestral e os dados são tomados como amostras para o cálculo dos custos totais de investimentos. O recorte temporal dos dados é o período de 2006 a 2016. Os dados usados nesse trabalho foram compilados por meio da base de dados Economática, acessada no dia 10/5/2017.

São selecionadas duas empresas da BOVESPA, de segmentos distintos, BRASKEN e RAIADROGASIL. A Tabela 1 traz as proxies empíricas usadas nos estudos do modelo funcional desenvolvido, encontradas em balanços patrimoniais e demonstrativos de resultados. Tais proxies são utilizadas na forma proposta por Aldrighi e Bisinha (2010), que realizam um estudo empírico usando dados de empresas listadas na BOVESPA com restrições financeiras. Conforme feito por aqueles autores, o valor da firma é tomado como o Enterprise value, uma medida que considera valor das ações, dívidas e participações dos acionistas minoritários: 
Enterprise value $=$ Valor de mercado das ações +

+ Dívida total líquida + Participação dos acionistas minoritários

O lucro operacional é calculado como a receita líquida de vendas e/ou serviços subtraída do custo de bens e/ou serviços vendidos. Este valor é anterior a tributações, pois se trata do resultado financeiro das vendas ou serviços prestados pela empresa. Também conforme Aldrighi e Bisinha (2010), o capital produtivo da empresa é aproximado apenas pelos seus ativos imobilizados, isto é, bens necessários para manutenção das atividades produtivas da empresa, a exemplo de maquinário e edificações. O investimento nestes bens, como prelecionam os referidos autores, é aproximado pela Capex (capital expenditure) diferida a cada período. Por fim, Aldrighi e Bisinha (2010) aplicam a razão entre Enterprise value e o ativo total para aproximar o $q$ de Tobin. Esta aproximação também é usada no presente estudo, conforme Tabela 1. Ressalta-se que a imprecisão das proxies selecionadas pode exacerbar os efeitos aleatórios dos erros das regressões, representados no modelo funcional pela Equação (12).

Finalmente, tomam-se por constantes as taxas r e $\delta$. É usada a taxa Selic de 2006, calculada para um trimestre em $r=4,06 \%$. Por sua vez, a taxa de depreciação $\delta$ varia com o maquinário que compõe o capital produtivo K e este texto limita-se a usar uma taxa genericamente definida para as empresas analisadas. Nota-se, pelos desenvolvimentos metodológicos anteriores, que a constante $\delta$ não altera a forma geral da curva de custos de ajuste de investimento. Portanto, não há perda de generalidade de resultados usando $\delta$ constante e igual para todas as empresas. Com isso fixa-se $\delta=1,68 \%$ como taxa de depreciação trimestral, seguindo as simulações registradas por Cooper e Haltiwanger (2006).

Tabela 1 - Proxies empíricas usadas no modelo teórico-funcional

\begin{tabular}{l|c|c}
\hline Nome & Valor no modelo & Proxie usada \\
\hline Valor da empresa & $V\left(K, \epsilon_{1}, \epsilon_{2}\right)$ & Enterprise value \\
\hline Lucro operacional & $\Pi\left(K, \epsilon_{1}\right)$ & Resultado bruto \\
\hline Estoque de capital produtivo & $K$ & Ativo Imobilizado \\
\hline Investimento & $I$ & Capex \\
\hline Preço sombra & $q$ & $\frac{\text { Enterprise value }}{\text { Ativo total }}$ \\
\hline
\end{tabular}




\section{Análise empírica}

Seguem resultados empíricos que ilustram curvas de custo de investimento e regimes de investimentos em função do $q$ de Tobin. Para tanto, admite-se que as empresas sempre escolhem o investimento ótimo da maximização na Equação (13) e, com isso, calculam-se os custos de investimento de acordo com a Equação (23) e os dados da Tabela 1. Conforme explicitado anteriormente, tratam-se os dados como amostras das variáveis independentes para o cálculo dos custos totais de ajustes de investimentos de forma instantânea no tempo. Para este fim, supõe-se que as curvas de custos de investimentos não variam com o tempo.

As equações dos custos totais de ajuste de investimentos são adaptadas a partir das variáveis independentes no lado direito da Equação (23), através de regressões lineares. São testadas duas formas empíricas para as curvas de ajuste de investimentos. A primeira forma é a quadrática, mais comum na literatura e explicitada na Equação (17). A segunda forma, proposta pelo presente texto, é a forma que admite uma componente de descontos por quantidade e custos fixos, dada na Equação (22). Os resultados sugerem, para as empresas selecionadas, que o uso de componentes de descontos aumenta a precisão das regressões com os dados empíricos apresentados. As regressões são avaliadas de acordo com as estatísticas $R^{2}$ e $F$ (não confundir com os custos fixos $F$ ) e com a significância dos coeficientes estimados. 
Tabela 2 - Custos de investimentos estimados por regressão linear na forma quadrática da Equação (18)

\begin{tabular}{|c|c|c|c|c|}
\hline \multicolumn{5}{|c|}{ BRASKEN } \\
\hline \multicolumn{5}{|c|}{ Coeficientes } \\
\hline & Estimativa & Erro padrão & valor t & valor-p \\
\hline$I^{2} / K$ & 0,5833 & 0,6125 & 0,952 & 0,347 \\
\hline \multicolumn{5}{|l|}{ Estatísticas } \\
\hline & $R^{2}$ & 0,02217 & $R^{2}$ ajustado & $-0,002275$ \\
\hline & $F$ & 0,9069 & valor-p & 0,3466 \\
\hline \multicolumn{5}{|c|}{ RAIADROGASIL } \\
\hline \multicolumn{5}{|c|}{ Coeficientes } \\
\hline & Estimativa & Erro padrão & valor t & valor-p \\
\hline$I^{2} / K$ & $1,120 \mathrm{e}-02$ & $4,029 e-03$ & 2,781 & 0,00928 \\
\hline Custo fixo $F$ & $1,012 e+10$ & $1,432 \mathrm{e}+10$ & 0,707 & 0,48510 \\
\hline \multicolumn{5}{|l|}{ Estatísticas } \\
\hline & $R^{2}$ & 0,2049 & $R^{2}$ ajustado & 0,1784 \\
\hline & $F$ & 7,733 & valor-p & 0,009276 \\
\hline
\end{tabular}

\subsection{Curvas de custos de investimentos}

A primeira regressão linear para as empresas BRASKEN e RAIADROGASIL busca adaptar o coeficiente $\gamma / 2$ das Equações (17) e (18). Os resultados são dados na Tabela 2. Apesar de esta ser a forma mais comumente assumida pela literatura, como estudado anteriormente, chama a atenção a pobre adaptação da curva ao caso da empresa BRASKEN. De fato, para a citada empresa, a curva não apresenta adaptação alguma aos dados, conforme aponta a estatística $R^{2}$. Por sua vez, a regressão quadrática aplicada à empresa RAIADROGASIL adapta-se melhor às variáveis, conforme esperado pela literatura. Para ilustrar o caso tratado por Abel e Eberly (1994), optou-se por incluir no caso RAIADROGASIL os custos fixos $F$, apesar de sua baixa significância, como observado pelo valor-p da regressão. A inclusão dos custos fixos $F$ neste caso não afeta a precisão da regressão, mas mantém a consistência com o caso teórico de Abel e Eberly (1994). 
As regressões lineares da Tabela 3 consideram a forma proposta na Equação (23) com $\eta=1.008$, usando investimentos positivos. Nota-se uma maior qualidade nas regressões para ambas as empresas, quando comparadas aos casos da Tabela 2. As comparações são feitas pelas estatísticas $R^{2}$ e $F$, bem como pelas as significâncias dos coeficientes nas respectivas tabelas. Portanto, fica demonstrada a maior capacidade de adaptação das curvas de custos totais de ajustes de investimentos aos casos em estudo com o uso de uma componente de descontos por quantidade.

Tabela 3 - Custos de investimentos estimados por regressão linear considerando descontos por quantidade, forma da Equação (23)

\begin{tabular}{|c|c|c|c|c|}
\hline \multicolumn{5}{|c|}{ BRASKEN } \\
\hline \multicolumn{5}{|l|}{ Coeficientes } \\
\hline & Estimativa & Erro padrão & valor $\mathrm{t}$ & valor-p \\
\hline$I^{2} / K$ & $1,932 e+00$ & $2,816 \mathrm{e}-01$ & 6,859 & $5,40 \mathrm{e}-07$ \\
\hline$I-0.008$ & $-1,903 e+13$ & $2,814 \mathrm{e}+12$ & -6.762 & $6,75 \mathrm{e}-07$ \\
\hline \multirow[t]{3}{*}{ Custo fixo $F$} & $1,650 e+13$ & $2,418 e+12$ & 6,824 & $5,86 \mathrm{e}-07$ \\
\hline & $R^{2}$ & 0,8986 & $R^{2}$ ajustado & 0,8898 \\
\hline & $F$ & 101,9 & valor-p & $3,71 \mathrm{e}-12$ \\
\hline \multicolumn{5}{|c|}{ RAIADROGASIL } \\
\hline \multicolumn{5}{|l|}{ Coeficientes } \\
\hline & Estimativa & Erro padrão & valor $t$ & valor-p \\
\hline$I^{2} / K$ & $7,452 \mathrm{e}-03$ & $2,984 \mathrm{e}-03$ & 2,497 & 0,02090 \\
\hline$I-0.008$ & $-3,687 e+12$ & $1,076 e+12$ & $-3,428$ & 0,00253 \\
\hline Custo fixo $F$ & $3,104 e+12$ & $8,965 e+11$ & 3,462 & 0,00233 \\
\hline \multicolumn{5}{|l|}{ Estatísticas } \\
\hline & $R^{2}$ & 0,6615 & $R^{2}$ ajustado & 0,6292 \\
\hline & $F$ & 20,52 & valor-p & $1,15 \mathrm{e}-05$ \\
\hline
\end{tabular}




\subsection{Retorno do investimento de acordo com o q de Tobin}

Conforme detalhado anteriormente, a medida $\psi\left(q, I^{*}, K\right)$ dada na Equação (24) permite analisar o retorno do investimento a partir do benefício marginal do mesmo. Tal benefício, medido pelo $q$ de Tobin, é buscado igualando-o ao custo marginal do investimento. Portanto a empresa busca um investimento ótimo $I^{*}$ sob a condição da Equação (16). Esta equação permite isolar a variável $I$ e obter a expressão da Equação (24) em função apenas de $q$ e $K$.

Ilustra-se o procedimento descrito acima com os resultados das regressões lineares da empresa RAIADROGASIL. No primeiro caso, de regressão quadrática da Tabela 2 , os custos do investimento são aproximados por $1,120 \cdot 10^{-2} I^{2} / K+1,012 \cdot 10 \cdot{ }^{10}$ Derivando esta expressão com relação a $I$ e igualando o resultado a $q$ conforme a Equação (16) tem-se $q=2,24$. $10^{-2} I / K$. Isolando $I$ e substituindo na Equação (24) chega-se a uma expressão em função de $q$ e $K$ :

$$
\psi(q, K)=\left[\frac{(1-r) K}{2,24 \cdot 10^{-2}}-22,32143 K\right] q^{2}+1,012 \cdot 10^{10}
$$

que tem raízes em $q=1,075194$ e $q=-1,075194$. Conforme descrito anteriormente, o capital produtivo $K$ nesta curva é fixado como a média dos ativos imobilizados durante todo o período estudado.

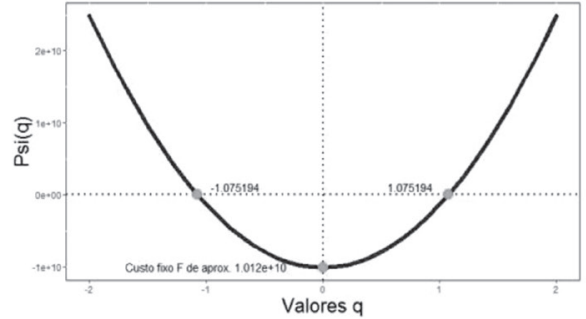

(a) Custos quadráticos

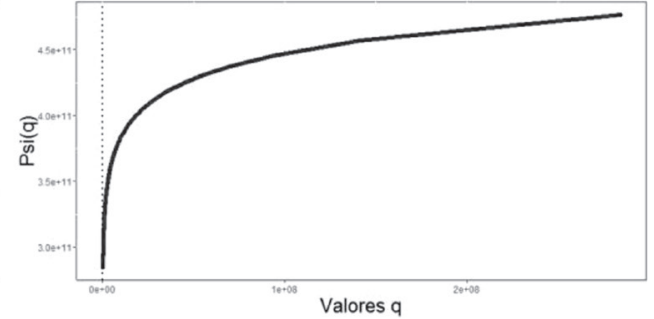

(b) Custos com descontos

Figura 1 - Medidas de retorno do investimento $\psi(q)$. Os cálculos são realizados através das Equações (24) e (16), usando as regressões lineares para aproximar os custos dos investimentos 
A Figura la ilustra a curva $\psi(q)$ calculada no parágrafo anterior. Trata-se de uma curva empírica que ilustra a forma apenas teórica proposta por Abel e Eberly (1994). Neste caso, a curva foi construída admitindo-se a forma dos custos de ajuste de investimento quadrática, como na Equação (17) e com a presença de custos fixos. Nesta figura são explícitas as raízes de $\psi(q)$ e o valor quando $q=0$, isto é, o custo fixo de aproximadamente $1,012 \cdot 10^{10}$. Sendo assim, para um capital de produção fixo na média dos valores no período de 2006 a 2016, o $q$ de Tobin deve ser superior a 1,075194 para um retorno positivo do investimento $I^{*}$ escolhido pela empresa RAIADROGASIL, nas condições propostas neste estudo.

A função $\psi(q)$ pode também ser derivada para o caso da curva de custo de ajuste de investimentos incluindo descontos por quantidade. Como exemplo, procede-se ao cálculo para a empresa RAIADROGASIL, com os dados da Tabela 3. O procedimento é o mesmo acima descrito para o caso quadrático e resulta na curva da Figura lb. Observa-se uma curva empírica bastante diferente da prevista por Abel e Eberly (1994). No caso de investimentos positivos da Figura lb, o comportamento assintótico da curva $\psi(q)$ sugere que a taxa de aumento do retorno do investimento é gradativamente menor conforme aumenta o $q$ de Tobin.

\section{Conclusões e recomendações}

Os custos de ajustes de investimento são parte integrante de estudos de valor da firma e investimentos em capital produtivo. Trata-se de custos incorridos em qualquer decisão de investimento e, portanto, devem ser computados para a valoração máxima da firma. Com isso, conhecer como variam e suas dependências torna-se fundamental.

A literatura de investimentos assume funções convexas e quadráticas para os custos de investimento. Além disso, assume-se que tais funções variem com os investimentos propriamente. Dessa forma, estudos e pesquisas podem desenvolver resultados analíticos com base em formas e funções de tratamento matemático geral. Contudo, os custos de investimento podem assumir dependências e formas diversas das comumente assumidas. 
Esta pesquisa demonstrou como funções de custos de investimento assumidas em textos anteriores adaptam-se a exemplos empíricos de empresas brasileiras. A aproximação quadrática de pesquisas anteriores não apresenta a precisão de outras formas aqui propostas. Importa registrar que a pesquisa aqui apresentada não especifica uma forma geral da componente de custos de ajuste na Equação (14) capaz de representar genericamente qualquer empresa. Os casos apresentados são aproximações possíveis, ilustradas com dados empíricos. Estudos futuros podem explorar uma forma geral desta componente, relacionando-a inclusive aos setores de atuação das empresas.

Investimentos reais estão sujeitos a negociações e preços diferenciados de fornecedores. Uma produtiva área de pesquisa em finanças de investimentos é a de descontos por quantidade. Este trabalho advoga que este assunto deve ser integrado às pesquisas de custos de investimento. Mesmo de forma simplificada, os descontos por quantidade foram evidenciados como parte integrante em custos de ajustes de investimento neste trabalho. Uma pesquisa mais profunda deve tomar modelos mais completos de descontos, considerando descontinuidades e incluindo os casos em que há desinvestimentos.

Não obstante algumas regressões lineares deste texto apresentarem alta precisão com relação aos valores reais obtidos das empresas, os custos de investimento encontram-se fortemente sujeitos a aleatoriedades. Os erros das regressões lineares não devem ser desprezados e por isso a função custo de ajuste de investimento deve ser sempre grafada em função de um processo estocástico. Cabe notar que os erros das equações podem ser maiores conforme imprecisões nas informações obtidas nos balanços patrimoniais e resultados das firmas.

Esta pesquisa deu forma prática às conclusões sobre retorno do investimento em função do $q$ de Tobin, da forma estudada por Abel e Eberly (1994). Aqueles autores analisaram uma situação convexa teórica de regimes de investimento em função do "preço sombra". Por sua vez, a pesquisa aqui realizada deu forma empírica às conclusões de Abel e Eberly (1994), confirmando seus resultados. Contudo tais resultados foram ainda estendidos a um outro exemplo, com forma diversa do trabalho original de Abel e Eberly (1994).

Registra-se aqui a limitação deste trabalho em assumir linearidade e homogeneidade para as funções de lucro operacional e custos de ajustes de investimentos. Conforme justificado no desenvolvimento do trabalho, essas 
assunções possibilitam estimar o $q$ de Tobin para as empresas estudadas, mas constituem restrições importantes. Portanto, outras formas de custos de investimento podem ser estudadas se descartada a limitação às funções linearmente homogêneas. Além disso, as curvas de ajustes de investimento são estudadas como invariáveis no tempo. Uma análise empírica mais abrangente pode examinar como tais curvas variam com o tempo, realizando um estudo longitudinal com dados em painel.

Por fim, um estudo mais geral de formas de custos de investimentos pode ser conduzido com dados empíricos de mais empresas. Além disso, sem as limitações citadas no parágrafo anterior, muitas adaptações analíticas poderiam ser empiricamente testadas em dados reais. Naturalmente seria necessário outro modelo para a estimativa dos valores $q$. Recomenda-se assim um estudo de medidas dos "preços sombra" sem fortes assunções sobre suas formas e funções mais gerais que as aqui tratadas para custos de ajuste de investimentos.

\section{Apêndice}

A Equação (7) pode ser reescrita como a soma de duas integrais, uma de $t$ a um tempo $\tau$ qualquer e outra de $\tau$ ao infinito:

$$
\begin{aligned}
V\left(K_{t}, \epsilon_{1, t}, \epsilon_{2, t}\right) & =\max _{I_{t}} \int_{t}^{\tau} E_{t}\left\{\Pi\left(K_{s}, \epsilon_{1, s}\right)-i\left(I_{s}\right)\left[C\left(I_{s}, K_{s}, \epsilon_{2, s}\right)+F_{s}-\xi\left(I_{s}\right)\right]\right\} e^{-r(s-t)} d s \\
& +\int_{\tau}^{\infty} E_{t}\left\{\Pi\left(K_{s}, \epsilon_{1, s}\right)-i\left(I_{s}\right)\left[C\left(I_{s}, K_{s}, \epsilon_{2, s}\right)+F_{s}-\xi\left(I_{s}\right)\right]\right\} e^{-r(s-\tau)} d s
\end{aligned}
$$

A segunda integral acima é o valor da firma no tempo $\tau$, portanto

$$
\begin{aligned}
V\left(K_{t}, \epsilon_{1, t}, \epsilon_{2, t}\right) & =\max _{I_{t}} \int_{t}^{\tau} E_{t}\left\{\Pi\left(K_{s}, \epsilon_{1, s}\right)-i\left(I_{s}\right)\left[C\left(I_{s}, K_{S}, \epsilon_{2, s}\right)+F_{s}\right.\right. \\
& \left.\left.-\xi\left(I_{s}\right)\right]\right\} e^{-r(s-t)} d s+E_{t}\left[V\left(K_{\tau}, \epsilon_{1, \tau}, \epsilon_{2, \tau}\right) e^{-r(\tau-t)}\right]
\end{aligned}
$$


Subtraindo ambos os lados da equação pela constante $V\left(K_{t}, \epsilon_{1, t}, \epsilon_{2, t}\right) e^{0} \mathrm{e}$ observando que $e^{-r(\tau-t)}=e^{-r \tau} e^{r t}$, bem como $e^{0}=e^{r t} e^{-r t}$, tem-se:

$$
\begin{aligned}
0=\max _{I_{t}} \int_{t}^{\tau} E_{t}\{ & \left.\Pi\left(K_{s}, \epsilon_{1, s}\right)-i\left(I_{s}\right)\left[C\left(I_{s}, K_{s}, \epsilon_{2, s}\right)+F_{s}-\xi\left(I_{s}\right)\right]\right\} e^{-r(s-t)} d s \\
+ & E_{t}\left[V\left(K_{\tau}, \epsilon_{1, \tau}, \epsilon_{2, \tau}\right) e^{-r \tau} e^{r t}-V\left(K_{t}, \epsilon_{1, t}, \epsilon_{2, t}\right) e^{r t} e^{-r t}\right]
\end{aligned}
$$

Colocando $e^{r t}$ em evidência e dividindo-se todos os termos por $\tau$, tem-se

$$
\begin{gathered}
\frac{0}{\tau}=\max _{I_{t}} \int_{t}^{\tau}\left(\frac{1}{\tau}\right) \\
E_{t}\left\{\Pi\left(K_{s}, \epsilon_{1, s}\right)-i\left(I_{s}\right)\left[C\left(I_{s}, K_{s}, \epsilon_{2, s}\right)+F_{S}-\xi\left(I_{s}\right)\right]\right\} e^{-r(s-t)} d s \\
+\left(\frac{E_{t}\left\{\left[V\left(K_{\tau}, \epsilon_{1, \tau}, \epsilon_{2, \tau}\right) e^{-r \tau}-V\left(K_{t}, \epsilon_{1, t}, \epsilon_{2, t}\right) e^{-r t}\right] e^{r t}\right\}}{\tau}\right)
\end{gathered}
$$

Uma vez que $\tau$ é arbitrário, pode-se fazê-lo suficientemente próximo a $t$ para expressar a equação em limites:

$$
\begin{aligned}
0=\max _{I_{t}} \lim _{\tau \rightarrow t} \int_{t}^{\tau}\left(\frac{1}{\tau}\right) E_{t}\left\{\Pi\left(K_{s}, \epsilon_{1, s}\right)-i\left(I_{s}\right)\left[C\left(I_{s}, K_{s}, \epsilon_{2, s}\right)+F_{s}-\xi\left(I_{s}\right)\right]\right\} e^{-r(s-t)} d s \\
+\lim _{\tau \rightarrow t}\left(\left(\frac{E_{t}\left\{\left[V\left(K_{\tau}, \epsilon_{1, \tau}, \epsilon_{2, \tau}\right) e^{-r \tau}-V\left(K_{t}, \epsilon_{1, t}, \epsilon_{2, t}\right) e^{-r t}\right] e^{r t}\right\}}{\tau}\right)\right)
\end{aligned}
$$

O primeiro limite pode ser imediatamente resolvido. Para simplificar o segundo limite, definem-se $X_{\tau}=V\left(K_{\tau}, \epsilon_{1, \tau}, \epsilon_{2, \tau}\right) e^{-r \tau}, X_{t}=V\left(K_{t}, \epsilon_{1, t}, \epsilon_{2, t}\right) e^{-r t}$ e $X=X_{\tau}-X_{t}$. Portanto

$$
0=\max _{I}\left\{\Pi\left(K, \epsilon_{1}\right)-i(I)\left[C\left(I, K, \epsilon_{2}\right)+F-\xi(I)\right]+\lim _{\tau \rightarrow t}\left(\frac{E_{t}[(X)] e^{r t}}{\tau}\right)\right\}
$$

O segundo limite acima pode ser resolvido observando-se que

$$
\frac{d X}{d t}=\frac{d}{d t}\left(X_{\tau}-X_{t}\right)=\frac{d}{d t}\left[V\left(K_{\tau}, \epsilon_{1, \tau}, \epsilon_{2, \tau}\right) e^{-r \tau}-V\left(K_{t}, \epsilon_{1, t}, \epsilon_{2, t}\right) e^{-r t}\right]
$$


e que:

$$
\frac{d X}{d t}=\frac{d}{d t}\left[\left(V_{\tau}-V_{t}\right) e^{-r t}\right]
$$

quando $\tau \rightarrow t$. Aplicando a regra da cadeia à equação acima resulta em

$$
\frac{d X}{d t}=\frac{d V}{d t} e^{-r t}-r X e^{-r t} \Rightarrow d X=d V e^{-r t}-(r X d t) e^{-r t}
$$

Finalmente observa-se que, no limite, $E_{t}(X)=E_{t}(d X)$ e aplicando a Equação (26) à Equação (25) resulta em

$$
0=\max _{I}\left\{\Pi\left(K, \epsilon_{1}\right)-i(I)\left[C\left(I, K, \epsilon_{2}\right)+F-\xi(I)\right]+\frac{E(d V)}{d t}-r V\left(K, \epsilon_{1}, \epsilon_{2}\right)\right\}
$$

Como $-r V\left(K, \epsilon_{1}, \epsilon_{2}\right)$ independe de $I$ na operação de maximização, tal termo pode passar ao lado esquerdo desta equação, resultando na forma de Bellman, usada na Equação (8) do texto. ${ }^{4}$

${ }^{4}$ O texto principal faz uso do valor presente da firma, sendo descontada portanto a esperança do valor futuro incremental $E(d V) / d t$ pela taxa $r$. 


\section{Referências}

Abel, A. B., e J. C. Eberly. 1994. “A Unified Model Of Investment Under Uncertainty.” The American Economic Review: 1369-1384.

Aldrighi, D. M., e R. Bisinha. 2010. "Restrição Financeira Em Empresas Com Ações Negociadas Na Bovespa." Revista Brasileira de Economia 64 (1): 25-47.

Barnett, S. A., e P. Sakellaris. 1998. "Nonlinear Response Of Firm Investment To Q::Testing A Model Of Convex And Non-Convex Adjustment Costs." Journal of Monetary Economics 42 (2): 261-288.

Bolton, P., H. Chen e N. Wang. 2011. “A Unified Theory Of Tobin’s Q, Corporate Investment, Financing, And Risk Management." The Journal of Finance 66 (5): 1545-1578.

Cooper, R. W., e J. C. Haltiwanger. 2006. "On The Nature Of Capital Adjustment Costs.” The Review of Economic Studies 73 (3): 611-633.

Gould, J. P. 1968. “Adjustment Costs In The Theory Of Investment Of The Firm." The Review of Economic Studies 35 (1): 47-55.

Groth, C., e H. Khan. 2010. "Investment Adjustment Costs: An Empirical Assessment”. Journal of Money, Credit and Banking 42 (8): 1469-1494.

Hamermesh, D. S., e G. A. Pfann. 1996. “Adjustment Costs In Factor Demand.” Journal of Economic Literature 34 (3): 1264-1292.

Hwang, H., D. H. Moon e S. W. Shinn. 1990. “An EOQ Model With Quantity Discounts For Both Purchasing Price And Freight Cost." Computers \& Operations Research 17 (1): 73-78.

Li, S. X., e Z. Huang. 1995. "Managing Buyer-Seller System Cooperation With Quantity Discount Considerations." Computers \& Operations Research 22 (9): 947-958.

Lundgren, T., e M. Sjöström. 2001. “A Flexible Specification Of Adjustment Costs In Dynamic Factor Demand Models." Economics Letters 72 (2): 145-150.

McDonald, R., e D. Siegel. 1986. "The Value Of Waiting To Invest.” The Quarterly Journal of Economics 101 (4): 707.

Munson, C. L., e M. J. Rosenblatt. 2009. “Theories And Realities Of Quantity Discounts: An Exploratory Study.” Production and Operations Management 7 (4): 352-369.

Pindyck, R. S. 1988. "Irreversible Investment, Capacity Choice, And The Value Of The Firm." The American Economic Review 78 (5): 969-985.

Rothschild, M. 1971. "On The Cost Of Adjustment." The Quarterly Journal of Economics 85 (4): 605-622.

Schotanus, F., J. Telgen e L. Deboer. 2009. “Unraveling Quantity Discounts.” Omega 37 (3): 510-521.

Tobin, J. 1969. "A General Equilibrium Approach To Monetary Theory." Journal of Money, Credit and Banking $1(1): 15$. 